\title{
Improved Promotion of M2 Microglial Polarization by Zuogui Jiangtang Jieyu Formulation in Diabetes- Related Depression
}

\section{xiuli zhang}

Hunan university of chinese medicine

\section{Dahua Wu}

Hunan Academy of Chinese Medicine: Hunan University of Chinese Medicine

Dandan Li

Hunan University of Chinese Medicine

Jian Liu

Hunan University of Chinese Medicine

Chang Lei

Hunan University of Chinese Medicine

\section{Yuehui Li}

Hunan Academy of Chinese Medicine: Hunan University of Chinese Medicine

\section{Lin Liu}

Hunan University of Chinese Medicine

\section{Dan Huang}

Hunan University of Chinese Medicine

Jinwen Ge ( $\nabla 001267 @ h n u c m . e d u . c n)$

Hunan University of Chinese Medicine

\section{Yuhong Wang}

Hunan University of Chinese Medicine

\section{Research}

Keywords: diabetes-related depression, microglial polarization, M1/M2 markers, inflammatory cytokines, ZGJTJY

Posted Date: March 23rd, 2021

DOI: https://doi.org/10.21203/rs.3.rs-335174/v1

License: (c) (1) This work is licensed under a Creative Commons Attribution 4.0 International License. 



\title{
Improved promotion of M2 microglial polarization by Zuogui Jiangtang Jieyu formulation in diabetes-related depression
}

\author{
Xiuli Zhang ${ }^{1,2}$, Dahua $\mathrm{Wu}^{5}$, Dandan $\mathrm{Li}^{3}$, Jian $\mathrm{Liu}^{4}$, Chang Lei ${ }^{1,2}$, Yuehui $\mathrm{Li}^{5}$, Lin $\mathrm{Liu}^{4}$, \\ Dan Huang ${ }^{1,2}$, Jinwen $\mathrm{Ge}^{3 *}$, Yuhong Wang ${ }^{1,2^{*}}$
}

\begin{abstract}
Background

Zuogui Jiangtang Jieyu formulation (ZGJTJY) is a Chinese polyherbal prescription for diabetes-related depression (DD). The mechanism underlying hippocampal M1/M2 polarization in $\mathrm{DD}$ and the ZGJTJY treatment effects remain unclear. This study aimed to investigate M1/M2 microglial polarization in the hippocampus of DD rats and HAPI (highly aggressively proliferating immortalized) cells simulating the DD state, as well as to examine the ZGJTJY intervention effects, both in vivo and in vitro.
\end{abstract}

\section{Methods}

We subjected Sprague Dawley rats to a high-fat diet, streptozotocin, and unpredictable chronic mild stress; subsequently, we orally administered ZGJTJY. HAPI cells were induced using high glucose and corticosterone; subsequently, ZGJTJY-containing serum was added to examine changes in M1/M2 microglial polarization. Moreover, metformin combined with fluoxetine (DMGB/F) was used as a positive drug for evaluating the ZGJTJY intervention. Laser confocal scanning was used to examine the microglial morphology. Further, real-time PCR was used to determine M1 markers (MHCII, iNOS, MCP-1, CD11b), M2 markers (Arg1, Mrc1, Ym1), pro-inflammatory cytokines (IL-1 $\beta$, IL-6, TNF- $\alpha$ ), and anti-inflammatory cytokines (IL-4, IL-10). Additionally, an enzyme-linked immunosorbent assay was used to examine inflammatory cytokines.

Results

There was significant activation of M1 polarization in the hippocampus of DD rats and HAPI cells induced using high glucose and corticosterone. Compared with DMGB/F, ZGJTJY inhibited and promoted M1 and M2 polarization, respectively; moreover, it decreased the M1-to-M2 polarization ratio both in vivo and in vitro.

\section{Conclusions}

The study indicated that hippocampal M1 polarization is crucially involved in DD pathogenesis; moreover, there is a need for further research on the neuroprotective effect of Chinese medicine associated with M2-polarized microglia.

Keywords: diabetes-related depression; microglial polarization; M1/M2 markers; inflammatory cytokines; ZGJTJY

*Correspondence: 001267@hnucm.edu.cnc, wyh107@126.com

*Jinwen Ge and Yuhong Wang contributed equally to this work.

${ }^{1}$ Hunan University of Chinese Medicine, No. 300, Xueshi Road, Hanpu Science and Education

Park, Yuelu District, Changsha 410208, China.

\section{Background}

According to the $9^{\text {th }}$ edition of the Global Diabetes Survey, there were approximately 463 million patients with diabetes in 2019 worldwide. Epidemiological studies have reported that compared with healthy individuals, the depression prevalence rate is $>3$ and $\approx 2$ times higher in patients with type 1 and 2 diabetes, respectively (Roy and Lloyd, 2012). Therefore, there is a need to elucidate the pathogenesis of diabetes-related depression (DD) to facilitate prevention and treatment.

Diabetes and depression could share common pathophysiological mechanisms; moreover, 
hippocampal pro-inflammatory cytokines are closely associated with the DD occurrence (da Silva Dias et al., 2016). Microglia are immune cells residing in the central nervous system (CNS), which are often the first responders to tissue damage or brain infections and are typically associated with inflammation. Under normal conditions, microglia are in the "resting" state and constantly surveil the surrounding environment (Nimmerjahn et al., 2005). Upon stimulation by pathological factors, microglia can be activated and polarized with resulting morphological and functional changes (Marshall et al., 2013). There are two types of activated microglia; namely, M1- and M2-polarized microglia. M1-polarized microglia may become amoeboid (Boche et al., 2013) and secrete pro-inflammatory cytokines, including IL-1 $\beta$, IL-6, and TNF- $\alpha$, which are involved in nerve injury (Réus et al., 2015). On the other hand, M2-polarized microglia release anti-inflammatory cytokines, including IL-4, IL-10, and TGF- $\beta$ (Ekdahl, 2012; Boche et al., 2013; $\mathrm{Hu}$ et al., 2015), which exert neuroprotective effects (Cherry et al., 2014). Therefore, M1 polarization could be directly involved in DD occurrence. Recent studies have shown that hyperglycemia and chronic stress can induce M1 polarization, which leads to obvious morphological changes, including swelling, cytoplasmic vacuoles, and excessive hypertrophy (Hwang et al., 2014; Zhao et al., 2016). However, antidiabetic drug or antidepressant administration can inhibit and promote M1 and M2 polarization, respectively (Darsalia et al., 2014; Kalkman and Feuerbach, 2016). Therefore, hippocampal M1 polarization may underlie DD pathogenesis. However, to our knowledge, there has been no study on the relationship between microglial polarization and DD.

In 2013, we successfully established a DD model using a 14-day high-fat diet (HFD), low-dose streptozotocin (STZ; $38 \mathrm{mg} / \mathrm{kg}$ ) injection, and 28-day chronic stress (Yang, 2013). To confirm the resulting DD model, the weight, as well as the levels of blood glucose, glycosylated hemoglobin, insulin, and blood lipids, were assayed. Plasma corticotropin-releasing hormone, adrenocorticotropic hormone, and corticosterone levels were measured. Hematoxylin and eosin staining was used to observe pathological changes in the hippocampus, hypothalamus, pituitary gland, and adrenal gland. Furthermore, the Morris water maze test, open field test, and forced swim test were performed. (Wang et al., 2015; 2014; Liu et al., 2018)

Traditional Chinese medicine (TCM) does not describe DD; however, diabetes and depression are referred to as "Xiao Ke" and "Yu" diseases, respectively. Based on TCM theory, DD shows the characteristics of TCM diagnoses of "Xu," "Yu," and "Yu" (Lin, et al., 2016). Accordingly, Zuogui Jiangtang Jieyu formulation (ZGJTJY) is prescribed based on the efficacy of ZuoguiWan, which was developed by Jingyue Zhang during the Ming dynasty and is widely used for treating diabetes mellitus and related complications (Wang, 2014; Liu et al., 2016). Based on TCM theory, ZGJTJY is comprised of five herbs in common with ZuoguiWan, including Rehmannia glutinosa (Gaertn.) Libosch. ex Fisch. \& C.A. Mey. (cooked root), Cornus officinalis Siebold \& Zucc. (fruit), L. barbarum L. (fruit), Cuscuta chinensis Lam. (seed), and Achyranthes bidentata Blume (root); moreover, it is comprised of Astragalus membranaceus (Fisch.) Bunge (Milkvetch Root) (root), Salvia miltiorrhiza Bunge (root), Hypericum perforatum L. (whole plant), and Curcuma longa L. (rhizome), which have been reported to inhibit depression by clinical studies (Di et al., 2017; Ruan and Ruan, 2006; Wang, 2014), as well as Paeonia suffruticosa Andrews (root bark) and Eucommia ulmoides Oliv. (bark). We previously showed that ZGJTJY could effectively improve glucose disorders, lipid metabolism, and depression symptoms in DD model rats, as well as reduce hippocampal damage and increase hippocampal synaptic remodeling (Wang et al., 2015; 2014; Zhang et al., 2015; Liu et al., 2018; Xiang et al., 2020). Moreover, we applied for a patent for ZGJTJY (No: 2019100732958) given its significant hypoglycemic and antidepressant effects. Although ZGJTJY could alter hippocampal microglial activation, it is yet to be studied.

This study aimed to examine microglial polarization in the hippocampus of DD rats and HAPI (highly aggressively proliferating immortalized) cells induced by high glucose and corticosterone. Moreover, we examined the ZGJTJY effect on microglial polarization and compared it with that of the combination of metformin and fluoxetine (DMGB/F). 


\section{Materials and Methods}

\subsection{ZGJTJY extract preparation}

ZGJTJY is composed of $18 \mathrm{~g} \mathrm{~g}$ A. membranaceus (Fisch.) Bunge (root), $3 \mathrm{~g}$ Hypericum perforatum L. (whole plant), $9 \mathrm{~g}$ Curcuma longa L. (rhizome), $15 \mathrm{~g}$ Rehmannia glutinosa (Gaertn.) Libosch. ex Fisch. \& C.A. Mey. (cooked root), 12 g Cornus officinalis Siebold \& Zucc. (fruit), 12 $\mathrm{g}$ of Lycium barbarum L. (fruit), $9 \mathrm{~g}$ Cuscuta chinensis Lam. (seed), and $9 \mathrm{~g}$ Eucommia ulmoides Oliv. (bark), $12 \mathrm{~g}$ Salvia miltiorrhiza Bunge (root), $6 \mathrm{~g}$ Paeonia suffruticosa Andrews (root bark), and $9 \mathrm{~g}$ Achyranthes bidentata Blume (root). Raw materials were purchased from the First Affiliated Hospital of Hunan University of Chinese Medicine and identified by Professor Dan Huang from Hunan University of Chinese Medicine, Changsha, China. Moreover, voucher specimens were deposited at the Technology Innovation Center, Hunan University of Chinese Medicine, Changsha, China. (specimen number: TCM-20180320). ZGJTJY was boiled, filtered, concentrated to a $2.28 \mathrm{~g} / \mathrm{ml}$ liquid at $80{ }^{\circ} \mathrm{C}$ in a water bath, and stored at $4{ }^{\circ} \mathrm{C}$ in a refrigerator. (Wang et al., 2014). For use, ZGJTJY was diluted with distilled water and administered by gavage.

\subsection{Other drugs and reagents}

We purchased metformin and fluoxetine hydrochloride tablets $(0.25 \mathrm{~g}$ and $20 \mathrm{mg}$, respectively) from Hunan Xiangya Pharmaceutical and Patheon France, respectively. The dose and HFD matching has been previously described (Wang et al., 2015). Pentobarbital sodium, corticosterone, STZ, and DAPI were obtained from Sigma (St Louis, MO, USA). Single-touch glucometers were obtained from LifeScan (High Wycombe, UK). D-glucose and bovine serum albumin (BSA) were obtained from Gibco (Grand Island, NY, USA). High-glucose Dulbecco's Modified Eagle Medium (DMEM) was obtained from Hyclone (Logan, UT, USA). Goat anti-Iba1 and donkey anti-goat IgG H\&L (FITC) were obtained from Abcam (Cambridge, UK). NoVoStart ${ }^{\circledR}$ SYBR qPCR SuperMix plus and TRIzol were obtained from NoVoprotein (Shanghai, China) and Takara (Kyoto, Japan), respectively. The Transcriptor First Strand cDNA Synthesis Kit was obtained from Roche (Indianapolis, USA). HAPI cells were obtained from Otwo Biotech Inc. (Shenzhen, China). ELISA kits for IL-1 $\beta$, IL-6, TNF- $\alpha$, IL-4, and IL-10 were obtained from MULTI SCIENCES (Hangzhou, China).

\subsection{Animal preparation and drug administration}

We purchased 24 male Sprague-Dawley rats $(200-220 \mathrm{~g})$ from Slack Scene of Laboratory Animal Company (Hunan, China) and kept them in the SPF Laboratory Animal Center in Hunan Chinese Medicine University. All procedures were approved by the Ethics Committee of Hunan University of Chinese Medicine (No: ZYFT20171206) and conducted according to the guidelines for the care and use of laboratory animals from the National Institutes of Health. Fig. 1 presents the flow chart of the study protocol.

The animals were housed in an SPF room $\left(22{ }^{\circ} \mathrm{C} \pm 3{ }^{\circ} \mathrm{C}, 50 \% \pm 5 \%\right.$ humidity, and a $12 / 12-\mathrm{h}$ circadian rhythm) with free access to water and diet. After 1 week of adaptive feeding, the rats were randomly divided into two groups. One group received a normal diet (containing $13.68 \%$ fat, $64.44 \%$ carbohydrate, and $21.88 \%$ protein) and citrate buffer once (i.v., the same volume as STZ). The other group received a HFD (p.o., $10 \%$ cholesterol, $0.2 \%$ propylthiouracil, $20 \%$ lard oil, $20 \%$ Tween 80 , and $20 \%$ propylene glycol to still water, $10 \mathrm{ml} / \mathrm{kg} / \mathrm{d})$ for 14 days and STZ once $(0.1$ $\mathrm{mol} / \mathrm{L}$ in citrate buffer [pH 4.5], $38 \mathrm{mg} / \mathrm{kg}$ i.v., fasting overnight before injection) at day 16 . After three days, rats with a fasting plasma glucose level $\geq 16 \mathrm{mmol} / \mathrm{L}$ were selected and randomized into three groups: vehicle, ZGJTJY, and DMGB/F groups ( $\mathrm{n}=6$ per group). Subsequently, the rats were subjected to 28 days of unpredictable chronic mild stress (UCMS), the Morris water maze test, open field test, and sucrose preference test for the successful establishment of the CUMS model as previously reported (Wang et al., 2014; 2015; Liu et al., 2019; Zhang et al., 2015). Simultaneously, $10.26 \mathrm{~g} / \mathrm{kg} /$ day ZGJTJY or a combination of 1.8 and $10.8 \mathrm{mg} / \mathrm{kg} /$ day DMGB and 
fluoxetine, respectively, were administered. The control group received an equal normal saline volume. A previous study showed that $10.26 \mathrm{~g} / \mathrm{kg} /$ day ZGJTJY was the most effective dose for improving DD model rats and their hippocampal pathological changes (Wang et al., 2015). DMGB and fluoxetine doses were calculated as human-to-rat equivalent doses based on the body surface areas.

On day 48 , rats were anesthetized using pentobarbital sodium (30 mg/kg, i.p.); subsequently, some whole brains were obtained after perfusion with saline and $4 \%$ paraformaldehyde (PFA) while the other brains were obtained to collect the hippocampus.

\subsubsection{Plasma glucose detection}

A single touch glucometer (One Touch Ultra 2; LifeScan, High Wycombe, UK) was used to determine glucose levels in plasma collected from the tail vein.

\subsubsection{Morris water maze test}

The Morris water maze was composed of a circular fiberglass pool (200 $\mathrm{cm}$ in diameter) filled with water $\left(25 \pm 1^{\circ} \mathrm{C}\right)$ and made opaque using black non-toxic paint. The pool was surrounded by light blue curtains fixed with three distal visual cues. Four floor light sources with equal power provided uniform illumination in the pool and testing room. A charge-coupled device camera (kl-9511zh, Konlan Company, Shuozhou, China) was suspended above the pool center to record the swim paths of the animals; further, the video output was digitized using an EthoVision XT tracking system (Noldus Information Technology, Inc., Leesburg, VA, USA).

Four trials in each quadrant were conducted once a day for five days. The video analysis system tracked, recorded, and analyzed the swimming speed and time taken to locate the platform for each animal. Each trial lasted until the rat located the platform or for $60 \mathrm{~s}$, with this time being recorded as the escape latency time (ELT); moreover, the learning outcome was the mean ELT for the last four days. On the final day, the platform was removed for a 60 -s probe trial, with the time spent swimming in the platform quadrant being recorded as the space exploration time (SET).

\subsubsection{Open field test}

The open-field device was an $80 \times 80 \mathrm{~cm}$ square box divided into 25 equilateral squares. The rat was placed in the central square; subsequently, we measured the number of squares crossed by the rat (only squares entered with all feet were included in the horizontal activity score) and the duration spent on hind limbs (the vertical activity score). Each rat underwent a 5-min test, which was scored by two observers with their average value being recorded. The sum of the horizontal and vertical activity scores was considered as indicative of the locomotor activity (LMA).

\subsubsection{Sucrose preference test}

Two bottles ( $1 \%$ sucrose solution vs. pure water) were individually presented to the rats for $24 \mathrm{~h}$. After adaptation, the rats fasted for $12 \mathrm{~h}$. Subsequently, both $1 \%$ sucrose solution and pure water were presented to rats for $15 \mathrm{~h}$, with measurement of the pre- and post-test sucrose intake volume. Sucrose preference was defined as the ratio of sucrose to the total weight (sucrose + water).

\subsection{Drug-containing serum preparation}

ZGJTJY and DMGB/F doses were calculated as thrice the effective dose in 2.3. Male Sprague-Dawley rats were randomly divided into the ZGJTJY (oral ZGJTJY administration at $30.78 \mathrm{~g} / \mathrm{kg} /$ day) and DMGB/F-treated groups (oral administration of metformin [5.4 mg/kg/day] and fluoxetine $[32.4 \mathrm{mg} / \mathrm{kg} / \mathrm{day}])$. Each treatment was administered twice daily for three days. One hour after the last intragastric administration, all the rats were anesthetized using an intraperitoneal injection of $10 \%$ chloral hydrate; moreover, blood was collected through the abdominal aorta under sterile conditions. Subsequently, the serum obtained was sterilized through filtering through a $0.22 \mu \mathrm{m}$ filter and stored at $-80^{\circ} \mathrm{C}$ until use. 


\subsection{Cell model preparation and intervention}

HAPI cells were cultured in high glucose DMEM ( $10 \%$ fetal bovine serum, $\left.5 \% \mathrm{CO}_{2}, 37{ }^{\circ} \mathrm{C}\right)$ followed by treatment with high glucose $(150 \mathrm{mM})$ and corticosterone $(200 \mu \mathrm{M})$ for in vitro simulation of the DD state, as previously reported (Liu et al., 2016; 2019). The control group was administered PBS. The ZGJTJY and DMGB/F groups were further administered with $10 \%$ volume of drug-containing serum while the control group received the corresponding serum. After fractionation, all the groups were tested for $24 \mathrm{~h}$.

\subsection{Immunofluorescence}

Brains were collected and fixed in 4\% PFA for $6-8 \mathrm{~h}$, followed by paraffin embedding and slicing into sections. After blocking using $10 \%$ normal goat serum for $1 \mathrm{~h}$ at room temperature, the brain slices were incubated with primary antibodies: goat anti-Iba1 (1:500) in $1 \%$ BSA at $4{ }^{\circ} \mathrm{C}$ overnight. After washing with PBS for $3 \times 5$ min, the slices were exposed to secondary antibodies: donkey anti-goat IgG H\&L (FITC) (1:1000) and DAPI (1: 1000) for $1 \mathrm{~h}$ at room temperature. Fluorescent images were captured using a confocal microscope (ZeissLSM800, Jena, Germany).

Cultured cells were fixed with 4\% PFA; subsequently, they were incubated with primary antibodies, secondary antibodies, and DAPI; moreover, immunofluorescence was detected through high-content analysis (PerkinElmer Operetta, Waltham MA, USA).

\subsection{Quantitative Real-Time PCR}

Total RNA was extracted from the hippocampus or cultured HAPI cells and isolated using TRIzol. We used a Transcriptor First Strand cDNA Synthesis Kit for cDNA synthesis. Real-time PCR was performed using the StepOne ${ }^{\mathrm{TM}}$ Real-Time PCR System (StepOne, Foster City, USA) with NoVoStart SYBR qPCR SuperMix plus. Table 1 presents the used primer sequences. The cycling conditions as follows: $95{ }^{\circ} \mathrm{C}$ for $5 \mathrm{~min}$ and 40 cycles of $95{ }^{\circ} \mathrm{C}$ for $10 \mathrm{~s}$, followed by $60{ }^{\circ} \mathrm{C}$ for $30 \mathrm{~s}$. Gene expression data were normalized using $\beta$-actin; moreover, relative gene expression levels were calculated using the $2^{-\Delta \Delta \mathrm{CT}}$ method.

Tab. 1. Primers for real-time PCR

\begin{tabular}{lll}
\hline Gene & SENS & REVS \\
\hline MHC II & AGGGCTCCCAGTGTCCTAG & GTCAGAGCAGGTTCAGGCAT \\
iNOS & ACACACAGCGCTACAACATC & GAAGGACTCTGAGGCTGTGT \\
MCP-1 & AGGTGTCCCAAAGAAGCTGT & ACAGAATGCTTGAGGTGGT \\
CD11b & GCAGCCAGATCGGCTCTTAT & CTAGGCAAGGGGCACACTG \\
IL-1 $\beta$ & TCCTCTGTGACTCGTGGGAT & TCACATGGGTCAGACAGCAC \\
IL-6 & AGAGACTTCCAGCCAGTTGC & CTGGTCTGTTGTGGGTGGTA \\
IL-12 & GTGACCCTCACCTGTGACAG & CCTTCTTGTGGAGCAGCAGA \\
TNF-a & CTGTGCCTCAGCCTCTTCTC & ACTGATGAGAGGGAGCCCAT \\
Arg1 & CCTATGCGTCATTTGGGTGG & TACACGATGTCCTTGGCAGA \\
Mrc1 & TGGTTGGATTGAGGCCTGAA & ATCCCATAAACCACCTGCCA \\
Ym1 & ATGGCCTCAACTTGGACTGG & GTGAGAAGCAGCCTTGGGAT \\
IL-4 & CCACGGAGAACGAGCTCATC & GAGAACCCCAGACTTGTTCTTCA \\
IL-10 & CATTCCATCCGGGGTGACAA & TGTTGTCCAGCTGGTCCTTC \\
TGF- $\beta$ & GGGCTTACCCCGGTAACAG & ATGGGGAGAACCAGATAAGGC \\
$\beta$-actin & AACCCTAAGGCCAACCGTGAAA & TCATGAGGTAGTCTGTCAGGTC \\
\hline
\end{tabular}

\subsection{Enzyme-linked immunosorbent assay (ELISA)}

ELISA was used to quantify pro-inflammatory (IL-1 $\beta$, IL-6, TNF- $\alpha$ ) and anti-inflammatory cytokines (IL-4 and IL-10) in the hippocampus or cultured HAPI cell supernatant. For supernatant collection, tissue samples were weighed, homogenized, and centrifuged at $4{ }^{\circ} \mathrm{C}$ for $10 \mathrm{~min}$ at 
$2,500 \mathrm{rpm}$.

\subsection{Statistical Analysis}

All statistical analyses were performed using SPSS 16.0 software (version 16.0, SPSS, Chicago, IL, USA). Results were presented as mean \pm standard error of the mean. Comparisons were performed using one-way analysis of variance, followed by a least significant difference test. Statistical significance was set as $P<0.05$.

\section{Results}

3.1 The DD model was successfully established using the combination of HFD, STZ, and UCMS, with ZGJTJY effectively improving the plasma glucose disorder and depression symptoms in DD model rats.

Compared with the control group, the vehicle group showed significantly higher plasma glucose levels $(P<0.05)$. In the Morris water maze test, compared with the control group, the vehicle group showed significantly longer ELTs on days 2,3 , and 4 , as well as significantly shorter SETs on day $5(P<0.05, P<0.01)$. In the open field test and sucrose preference test, compared with the control group, the vehicle group showed lower LMA scores and sucrose preference (Tab.2)

Compared with the vehicle group, both DMGB/F and ZGJTJY reduced blood glucose, shortened EL times, increased total LMA scores, and increased sucrose intake. (Tab.2)

Fig. 1. The flow chart of the study protocol

Tab. 2 Capability of learning and memory, locomotor activity, sucrose preference, and plasma glucose level in each group

\begin{tabular}{|c|c|c|c|c|c|c|c|c|}
\hline \multirow{2}{*}{ Group } & \multicolumn{4}{|l|}{ ELT(s) } & \multirow{2}{*}{ SET(s) } & \multirow{2}{*}{ LMA(score) } & \multirow{2}{*}{$\begin{array}{l}\text { Sucrose } \\
\text { preference }\end{array}$} & \multirow{2}{*}{$\begin{array}{l}\text { Serum } \\
\text { glucose(mM) }\end{array}$} \\
\hline & Day 1 & Day2 & Day 3 & Day 4 & & & & \\
\hline Control & $50.02 \pm 1.25$ & $39.45 \pm 2.27$ & $21.34 \pm 3.35$ & $9.76 \pm 3.21$ & $25.13 \pm 0.25$ & $38.23 \pm 0.65$ & $0.82 \pm 0.06$ & $5.88 \pm 0.72$ \\
\hline Vehicle & $59.38 \pm 1.38$ & $59.27 \pm 3.08^{*}$ & $56.43 \pm 3.51^{*}$ & $54.44 \pm 1.01^{* *}$ & $18.23 \pm 0.24^{*}$ & $5.06 \pm 2.32^{* *}$ & $0.54 \pm 0.07^{* *}$ & $29.45 \pm 1.08^{* * *}$ \\
\hline ZGЛTJY & $56.25 \pm 1.45$ & $52.12 \pm 2.35$ & $43.78 \pm 2.26$ & $34.87 \pm 2.11^{\#}$ & $21.26 \pm 0.38$ & $15.45 \pm 1.29^{\sharp}$ & $0.69 \pm 0.07^{\#}$ & $18.72 \pm 1.78^{\#}$ \\
\hline DMGB/F & $54.22 \pm 3.23$ & $50.41 \pm 2.42$ & $40.35 \pm 2.08^{\#}$ & $32.57 \pm 2.48^{\#}$ & $23.34 \pm 0.31^{\#}$ & $20.52 \pm 1.07^{\#}$ & $0.76 \pm 0.08^{\#}$ & $15.45 \pm 1.29^{\#}$ \\
\hline
\end{tabular}

${ }^{*} P<0.05$ and ${ }^{* *} P<0.01$, significantly different from control. ${ }^{\sharp} P<0.05$ and ${ }^{\# \#} P<0.01$, significantly different from the vehicle group, $\mathrm{n}=6$.

3.2 The combination of HFD, STZ, and UCMS induced significant changes in microglial morphology and quantity in the hippocampal CA1 and DG areas. Unlike DMGB/F, which only acted on the CA1 area, ZGJTJY reversed these abnormalities in the CA1 and DG areas.

In the CA1 area of the vehicle group, there was cell body expansion, shortening and thickening of synapses, and a decreased microglial number. Both ZGJTJY and DMGB/F reversed these changes by ameliorating the cell body swelling, as well as promoting the increase, lengthening, and thinning of synaptic branches. Compared with DMGB/F, ZGJTJY appeared to have a more obvious effect on promoting cell body retraction and microglial proliferation. (Fig. 2).

The vehicle group showed similar microglial changes in the DG area, which were alleviated by ZGJTJY, but not DMGB/F, administration (Fig. 2).

Fig. 2. Immunofluorescence staining of Ibal (green) to detect microglial activation in the hippocampal CA1 and DG areas, with DAPI staining is shown in blue (200 x). "Resting" microglia in the control group. (A) The vehicle group showed activated microglia with cell expansion, thicker branching, and a decreased cell number (B). DMGB/F reversed abnormalities in the CA1, but not DG, area. (C) ZGJTJY reversed these abnormalities in both the CA1 and DG areas (D), $\mathrm{n}=3$. 
3.3 The combination of HFD, STZ, and UCMS induced different changes in M1/M2 microglia markers and inflammatory cytokines in the hippocampus; ZGJTJY reduced M1 polarization while up-regulating M2 polarization.

The combination of HFD, STZ, and UCMS increased the expression of M1 markers (MHCII, iNOS, MCP-1, CD11b) (Fig. 3A-D, $P<0.01$ ) and pro-inflammatory cytokines (IL-1 $\beta$, IL-6, TNF- $\alpha$ ) (Fig. 4 A-C, Fig. 5A-C, $P<0.01$ ); however, it decreased the expression of M2 marker Ym1 (Fig. 3G, $P<0.05$ ) and anti-inflammatory cytokines IL-4 (Fig. 4D, $P>0.05$; Fig. 5D, $P<$ 0.01 ) and IL-10 (Fig. 4E, $P<0.01$; Fig. 5E, $P>0.05$ ), except Mrc1 (Fig. 3F).

ZGJTJY administration decreased the expression of M1 markers MHCII, MCP-1, and CD11b (Fig. 3A, C, D, $P<0.01$ ); iNOS (Fig. 3B, $P>0.05$ ); and pro-inflammatory cytokines IL-1 $\beta$, IL-6, and TNF- $\alpha$ (Fig. 4A-C, Fig. 5A-B, $P<0.01$; Fig. 5C, $P>0.05$ ); and iNOS (Fig. 3 A-D, Fig. $4 \mathrm{~A}-\mathrm{C}$, Fig. $5 \mathrm{~A}-\mathrm{C}, P<0.01)$.

ZGJTJY significant increased the expression of M2 markers Arg1, Mrc1, Ym1 (Fig. 3E-G, $P$ $<0.01$ ), as well as anti-inflammatory cytokines IL-10 and IL-4 (Fig. 4D, E, Fig. 5D, E, $P<0.01$, or $P<0.05)$. DMGB/F had similar effects on $\mathrm{M} 2$ markers and anti-inflammatory cytokines as ZGJTJY (Fig. 3E, G, Fig. 4D, E, $P<0.01$; Fig. 5D, E, $P<0.01$, or $P<0.05$; Fig. 3F, $P>0.05$ ).

3.4 Compared with DMGB/F, ZGJTJY could better promote M2 polarization, as well as alter the ratio between $\mathrm{M} 1$ and $\mathrm{M} 2$ polarization in vivo.

Compared with DMGB/F, ZGJTJY had better inhibition of MCP-1, CD11b (Fig. 3C, D, $P<0.05$ or $P<0.01$ ), and IL-1 $\beta$ (Fig. 4A, Fig. 5A, $P<0.01$ ); however, it showed worse inhibition of MHC II, iNOS (Fig. 3A, B, $P<0.01$ or $P>0.05$ ), and IL-6 (Fig. 4B, Fig. 5B; $P<0.01$ or $P<0.05$ ).

Compared with DMGB/F, ZGJTJY improved the expression of M2 markers Arg1, Mrc1, and Ym1 (Fig. 3E-G, $P<0.01$, or $P<0.05$ ), as well as anti-inflammatory cytokines (Fig. 4D, E, $P<$ 0.01 ; Fig. 5D, E, $P<0.01$, or $P<0.05$ ).

ZGJTJY administration significantly decreased the ratio of MCP-1/Mrc1(Fig. $3 \mathrm{H}, P<0.01$ ) and IL-1 $\beta / \mathrm{IL}-4$ (Fig. 4F, Fig. 5F, $P<0.01$ ); moreover, compared with DMGB/F, it showed a stronger capacity to reduce the ratio between $\mathrm{M} 1$ and M2 polarization (Fig. 3H, Fig. 4F, Fig. 5F; $P$ $<0.01)$.

Fig. 3. mRNA expressions of hippocampal M1/M2 microglia markers. Fold increase of M1 markers (MHCII, iNOS, MCP-1, CD11b) (A, B, C, D); Fold increase of M2 microglia markers (Arg1, Mrc1, Ym1) (E, F, G); Fold increase of the ratio of MCP-1/Mrcl (H). Each bar represents the mean \pm SD of three samples. ${ }^{*} P<0.05$ and ${ }^{* *} P<$ 0.01 , significantly different from the control group. ${ }^{\#} P<0.05$ and ${ }^{\#} P<0.01$, significantly different from the vehicle group. ${ }^{\Delta} P<0.05$ and ${ }^{\Delta \Delta} P<0.01$, significantly different from the DMGB/F group.

Fig. 4. mRNA expressions of different hippocampal inflammatory cytokines. Fold increase of pro-inflammatory cytokines (IL-1 $\beta$, IL-6, TNF- $\alpha$ ) (A, B, C); M2 anti-inflammatory cytokine (IL-4, IL-10) (D, E); and IL-1 $\beta /$ IL-4 (F). Each bar represents the mean \pm SD of three samples. ${ }^{*} P<0.05$ and ${ }^{* * *} P<0.01$, significantly different from the control group. ${ }^{\#} P<0.05$ and ${ }^{\# \#} P<0.01$, significantly different from the vehicle group. ${ }^{\Delta} P<0.05$ and ${ }^{\Delta \Delta} P<0.01$, significantly different from the DMGB/F group.

Fig. 5. ELISA analysis of different hippocampal inflammatory cytokines. Levels of M1 pro-inflammatory (IL-1 IL-6, TNF- $\alpha$ ) (A, B, C) and anti-inflammatory cytokines (IL-4, IL-10) (D, E). The ratio of IL-1ß/IL-4 (F). Each bar represents the mean \pm SD of three samples. ${ }^{\#} P<0.05$ and ${ }^{\# \#} P<0.01$, significantly different from the vehicle group. ${ }^{\Delta} P<0.05$ and ${ }^{\Delta \Delta} P<0.01$, significantly different from the DMGB/F group.

3.5 High glucose and corticosterone induced microglial activation similar to the DD state, which was attenuated by ZGJTJY-containing serum in vitro. 
Further, high glucose combined with corticosterone induced microglial cell expansion, increased branching thickness, and decreased cell number (Fig. 6).

The combination of high glucose and corticosterone increased M1 markers $(P<0.01)$; MHC II, iNOS, MCP-1, and CD11b (Fig. 7A-D, $P<0.01$ ); and pro-inflammatory cytokines IL-1 $\beta$, IL-6, and TNF- $\alpha$ (Fig. 8A-C, Fig. 9A-C, $P<0.01$ ), which was similar to the STZ- and UCMS-induced effect on microglia in DD rats. ZGJTJY- and DMGB/F-containing serum both reversed microglial abnormalities (Fig. 7A-D, Fig. 8A-C, $P<0.01$; Fig. 9A-C, $P<0.01$, or $P<0.05$ ).

Additionally, ZGJTJY and DMGB/F administration significantly increased the expression of M2 markers Arg1, Mrc1, and Ym1(Fig. 7E-G, $P<0.01$, or $P<0.05$ ), as well as anti-inflammatory cytokines IL-10, IL-4, and TGF- $\beta$ (Fig. 8D, E, Fig. 9D, E, $P<0.01$, or $P<$ $0.05)$.

\subsection{In vitro, ZGJTJY-containing serum showed better promotion of M2 polarization and alteration of the ratio between M1 and M2 polarization.}

Compared with DMGB/F-containing serum, ZGJTJY-containing serum had a stronger inhibitory effect on MCP-1 (Fig. 7C, $P<0.01$ ) and IL-1 $\beta$ (Fig. 8A, $P<0.01$; Fig. 9A, $P>0.05$ ); however, it showed a weaker inhibitory effect on iNOS, CD11b (Fig. 7B, D, $P<0.05$ ), and IL-6 (Fig. 8B, Fig. 9B, $P<0.01$, or $P<0.05$ ).

Additionally, compared with DMGB/F-containing serum, ZGJTJY-containing serum could better promote M2 markers (Fig. 7E-G, $P<0.01$ ) and anti-inflammatory cytokines (Fig. 8D, E, $P$ $<0.01$; Fig. 9D, E, $P<0.05$ ), as well as reduce the ratio of MCP-1/Mrc1 (Fig. $7 \mathrm{H}, P<0.01$ ) and IL-1 $/$ IL-4(Fig. 8 F, $P<0.01$; Fig. 9F, $P<0.05$ ).

Fig. 6. Immunofluorescence staining of Iba1(green) for detecting microglial activation induced by high glucose and corticosterone, with DAPI staining shown in blue(200x). HAPI cells in the control group (A). Activated microglia with cell expansion, thickening of branching, and decreased cell number in the Vehicle group (B). DMGB/F- (C) and ZGJTJY-containing serum(D) reversed these abnormalities.

Fig. 7. mRNA expressions of M1/M2 microglia markers in HAPI cells. Fold increase of M1 markers (MHCII, iNOS, MCP-1, CD11b) (A, B, C, D); M2 microglia markers (Arg1, Mrc1, Ym1) (E, F, G); and MCP-1/Mrc1 ratio (H). Each bar represents the mean \pm SD of three wells. ${ }^{*} P<0.05$ and ${ }^{* *} P<0.01$, significantly different from the control group. ${ }^{\#} P<0.05$ and ${ }^{\#} P<0.01$, significantly different from the vehicle group. ${ }^{\Delta} P<0.05$ and ${ }^{\Delta \Delta} P<0.01$, significantly different from the DMGB/F group.

Fig. 8. mRNA expressions of different inflammatory cytokines in HAPI cells. Fold increase of pro-inflammatory

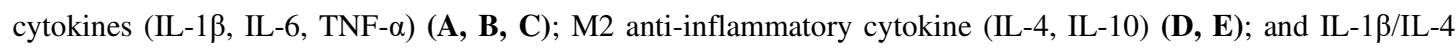
ratio $(\mathbf{F})$. Each bar represents the mean \pm SD of three wells. ${ }^{*} P<0.05$ and ${ }^{* *} P<0.01$, significantly different from the control group. ${ }^{\#} P<0.05$ and ${ }^{\#} P<0.01$, significantly different from the vehicle group. ${ }^{\Delta} P<0.05$ and ${ }^{\Delta \Delta} P<$ 0.01 , significantly different from the DMGB/F group.

Fig. 9. ELISA analysis of different inflammatory cytokines in cultured HAPI cell supernatant. Content of pro-inflammatory (IL-1 $\beta$, IL-6, TNF- $\alpha)(\mathbf{A}, \mathbf{B}, \mathbf{C})$ and M2 anti-inflammatory cytokines (IL-4, IL-10) (D, E). The ratio of IL-1 $\beta / \mathrm{IL}-4$ (F). Each bar represents the mean \pm SD of three wells. ${ }^{\#} P<0.05$ and ${ }^{\#} P<0.01$, significantly different from the vehicle group. ${ }^{\Delta} P<0.05$ and ${ }^{\Delta \Delta} P<0.01$, significantly different from the DMGB/F group.

\section{Discussion}

This study confirmed that the combination of HFD, STZ, and CUMS could induce M1 microglial polarization and increase hippocampal pro-inflammatory cytokines. Moreover, we found that the combination of high glucose and corticosterone induced microglial polarization similar to the DD state in vitro. Additionally, we found that ZGJTJY administration reversed these abnormalities by inhibiting and promoting M1 and M2 polarization, respectively. Compared with DMGB/F, ZGJTJY had no significant advantage in downregulating M1 polarization; however, it allowed better promotion of $\mathrm{M} 2$ polarization. These findings may help provide insight for research on the mechanism underlying TCM treatment of neuropsychiatric diseases. 
We have previously established the rat model of DD (Yang, 2013; Wang et al., 2014; 2015). The combination of HFD, STZ, and CUMS could induce high blood glucose levels, increase insulin sensitivity, and depressive-like behaviors, which mimicked the DD clinical features. The model establishment methods were reliable and stable. da Silva Dias et al. (2016) established a DD model by injecting STZ into overnight fasted rats. Compared with this method, the chronic mild stress used in our research can better simulate the daily-life living pressure.

In vitro, LPS and IL-4 are commonly used to induce M1 and M2 microglial polarization, which is related to depression, diabetes mellitus (Guo et al., 2019; Zhang et al., 2015), and other neuroinflammation-related diseases (Jung et al., 2016; Yang, et al., 2019). Our previous study (Wang et al., 2015) and other studies (Ding et al., 2018; Lebedeva, et al., 2017; Jimeno et al., 2018) have shown that high corticosterone levels induced by a handling and restraint stressor could increase blood glucose levels, which causes depressive behaviors. Moreover, hippocampal corticosterone accumulation contributes to hippocampal damage in DD. Hsieh et al. (2019) reported that acute glucose fluctuation induces stress, which alters microglial polarization. Furthermore, we used a combination of high glucose and corticosterone to induce primary hippocampal neuron damage, similar to the DD state (Zhang et al., 2014). We recently established a hippocampal NVU system comprised of a triple-cell co-culture system (brain microvascular endothelial cells, astrocytes, and neurons) in vitro using a combination of high glucose and corticosterone (Liu et al., 2019). In this study, M1 microglial polarization was induced by high glucose and corticosterone levels. This yielded a cell model of microglial polarization that could better reflect the physiological characteristics of DD; moreover, it confirmed that M1 microglial polarization caused by high corticosterone levels is closely associated with hippocampal damage.

Previous studies have reported that the monoaminergic system, hypothalamic-pituitaryadrenal axis, neuro-inflammation, and hippocampal damage are involved in DD pathogenesis (Zanoveli et al., 2016; da Silva Dias et al., 2016; Wang et al., 2015). However, the biological mechanism underlying this relationship remains unclear. Several studies have reported that M1 microglial polarization can directly inhibit hippocampal nerve regeneration (Michelle, et al., 2003), weaken the hippocampal neuroprotective effect (Patterson, 2015), and induce neurotoxicity (Ekdahl, et al., 2009) through inflammatory cytokine release. Meanwhile, microglia can be repeatedly activated, which causes multiple neuronal trauma by acting with astrocytes and microvascular cells (Steelman et al., 2014; Takaki et al., 2012; da Fonseca et al., 2014). Further, it can reduce 5-HT synthesis by activating the indoleamine 2,3-dioxygenase enzyme, which is the first rate-limiting enzyme in 5-HT precursor tryptophan metabolism (Xie et al., 2014); moreover, it promotes Glu release from astrocytes, which results in excitatory poisoning of the central nervous system by binding to NMDA receptors (Steiner et al., 2011). Moreover, the released neurotoxic media can act as microglia, deepen microglial polarization, aggravate neuronal damage, and form a vicious circle (Barger et al., 2007;). Therefore, we speculated that under the continuous hyperglycemic stress, continuous activation of hippocampal M1 microglia can cause severe hippocampal damage. This suggests that M1 polarization arising from hippocampal corticosterone accumulation can underlie DD pathogenesis.

ZGJTJY has been shown to reduce hippocampal corticosterone expression and increase glucocorticoid receptor (GR) expression in DD rats (Wang et al., 2015). GR has been shown to have a negative regulatory effect on M1 polarization (Réus et al., 2015). It is involved in microglial differentiation, proliferation, and motility; moreover, it is related to TLR4 (Carrillo-de Sauvage et al., 2013). TLR4 signaling pathway activation is involved in promoting M2 microglia polarization (Yang et al., 2019; Tian et al., 2019). Therefore, we suggest that the interaction of ZGJTJY with microglial polarization is dependent on corticosterone/GR/TLR4 signaling.

M1/M2 microglial polarization is crucially involved in the balance between inflammation promotion and suppression (Tao et al., 2016). Transition to the M1 and M2 phenotypes causes neurotoxicity and neuroprotective effects, respectively (Cherry et al., 2014). The ratio between M1 and M2 polarization reflects the transition from M1 to M2 activation. In this study, ZGJTJY 
administration reduced the ratio of MCP-1/Mrc1 and IL-1ß/IL-4, which induced M1-to-M2 transition.

This study has several limitations. First, we only assessed the hippocampal CA1 and DG areas, which cannot represent the global hippocampal microglia polarization. However, we selected these two areas because a previous study reported a predominant increase in IL-1 $\beta$ immunoreactivity in the hippocampal CA1 and DG areas (Hwang et al., 2014). Consistently, neuron proliferating disorder has been observed in the DG and CA1 areas of type-2 diabetes (Hwang et al., 2008). These findings implicate the hippocampal CA1 and DG areas in the interaction between diabetes and depression. Second, we only detected hippocampal microglial polarization and did not examine nerve apoptosis and regeneration in the CA1 and DG areas. Third, we did not screen the active ingredients of the ZGJTJY formulation that acted on microglial polarization.

\section{Conclusion}

Our findings suggest that DD is associated with an enhanced pro-inflammatory M1/M2 microglial ratio both in vivo and in vitro. ZGJTJY administration inhibited microglial polarization to a pro-inflammatory state and promoted an anti-inflammatory state by decreasing the M1/M2 microglial ratio. Future interventions on neuroinflammation-related neuropsychiatric diseases should target both prevention and promotion of M1 and M2 microglial polarization, respectively.

\section{Abbreviations}

DD, diabetes-related depression; TCM, traditional Chinese medicine; ZGJTJY, Zuogui Jiangtang Jieyu formulation; DMGB/F, metformin combined with fluoxetine; STZ, streptozotocin; CUMS, chronic unpredictable mild stress; MHCII, major histocompatibility complex; iNOS, induced nitric oxide synthase; MCP-1, monocyte chemoattractant protein-1; Arg1, Arginase1; Mrc1, mannose receptor $\mathrm{C}$ type 1; Ym1, Chitinase-3-like protein 3.

\section{Ethics approval and consent to participate}

Not applicable

\section{Consent for publication}

Not applicable.

\section{Availability of data and materials}

The datasets generated and/or analyzed during the current study are available from the corresponding author on reasonable request.

\section{Competing interests}

The authors declare no conflicts of interest.

\section{Funding}

This work was supported by the National Natural Science Foundation of China (No. 81603608, 81874462), Key Research and Development Program of Hunan Province (No. 2020SK2123), Natural Science Project of Hunan Province (No.2019JJ50431), and Open Fund for Top Discipline of Traditional Chinese Medicine of Hunan University of Chinese Medicine (No. 4901-020000200221).

\section{Authors' contributions}

J. G., Y. W., and X. Z. designed the project. P. M. and D. L. established an animal model. J. L. performed laser confocal scanning. L. L. and X. Z. performed the real-time PCR and cell culture. C.L. performed the drug preparation, analysis, and quality control. X. Z. wrote the manuscript. All the authors discussed and commented on the manuscript. 


\section{Acknowledgments}

Not applicable.

\section{Authors' information}

${ }^{1}$ Technology Innovation Center, Hunan University of Chinese Medicine, Changsha, Hunan410208, China. ${ }^{2}$ Hunan Key Laboratory of Powder and innovative drugs State Key Laboratory of ministry training bases, Changsha, Hunan 410208, China. ${ }^{3}$ Key Laboratory of Hunan Province for Prevention and Treatment of Integrated Traditional Chinese and Western Medicine on Cardiocerebral Diseases, Hunan University of Chinese Medicine, Changsha, Hunan 410208, China. ${ }^{4}$ First Hospital of Hunan University of Chinese Medicine, Changsha, Hunan 410208, China. ${ }^{5}$ Hunan Academy of Chinese Medicine, Changsha, Hunan 410208, China.

\section{References}

Barger, S.W., Goodwin, M.E., Porter, M.M., Beggs, M.L., 2007. Glutamate release from activated microglia requires the oxidative burst and lipid peroxidation. J Neurochem. 101, 1205-1213. https://doi.org/10.1111/ j. 147-4159.2007.04487.x

Boche, D., Perry, V.H., Nicoll, J.A., 2013. Review: activation patterns of microglia and their identification in the human brain. Neuropathol Appl Neurobiol. 39, 3-18. https://doi.org/10.1111/nan.12011

Carrillo-de Sauvage, M.Á., Maatouk, L., Arnoux, I., Pasco, M., Sanz Diez, A., Delahaye, M., Herrero, M.T., Newman, T.A., Calvo, C.F., Audinat, E., Tronche, F., Vyas, S., 2013. Potent and multiple regulatory actions of microglial glucocorticoid receptors during CNS inflammation. Cell Death Differ. 20, 1546-1557. https://doi.org/10.1038/cdd.2013.108

Cherry, J.D., Olschowka, J.A., O'Banion, M.K., 2014. Neuroinflammation and M2 microglia: The good, the bad, and the inflamed. J Neuroinflammation. 11, 98. https://doi.org/10.1186/1742-2094-11-98

da Fonseca, A.C., Matias, D., Garcia, C., Amaral, R., Geraldo, L.H., Freitas C., Lima, F.R., 2014. The impact of microglial activation on blood-brain barrier in brain diseases. Front Cell Neurosci. 8, 1-13. https://doi.org/10.3389/fncel.2014.00362

da Silva Dias, I.C., Carabelli, B., Ishii, D.K., de Morais, H., de Carvalho, M.C., Rizzo de Souza, L.E., Zanata, S. M., Brandão, M.L., Cunha, T.M., Ferraz, A.C., Cunha, J.M., Zanoveli, J.M., 2016. Indoleamine-2,3-dioxygenase/kynurenine pathway as a potential pharmacological target to treat depression associated with diabetes. Mol Neurobio. 53, 6997-7009. https://doi.org/10.1007/s12035-015-9617-0

Darsalia, V., Hua, S., Larsson, M., Mallard, C., Nathanson, D., Nyström, T., Sjöholm, Å., Johansson, M.E., Patrone, C., 2014. Exendin-4 reduces ischemic brain injury in normal and aged type 2 diabetic mice and promotes microglial M2 polarization. PLoS One. 9, e103114. https://doi.org/10.1371/journal.pone.0103114

Ding, H., Cui, X.Y., Cui, S.Y., Ye H., Hu X., Zhao H.L., Liu, Y.T., Zhang, Y.H., 2018. Depression-like behaviors induced by chronic corticosterone exposure via drinking water: Time-course analysis. Neurosci Lett. 687, 202-206. https://doi.org/10.1016/j.neulet.2018.09.059.

Di, W.J., Wang, D.H., Zhou, X.Q., 2017. Effect of Astragalus injection combined with Danshen Injection on depression after cerebral infarction. $J$ Tradit Chin Med. 33, 130-131. https://doi.org/10.3969/j.issn.1004-2814.2017.02.015

Ekdahl, C.T., Kokaia, Z., Lindvall, O., 2009. Brain inflammation and adult neurogenesis: The dual role of microglia. Neuroscience. 158, 1021-1029. https://doi.org/10.1016/j.neuroscience.2008.06.052

Ekdahl, C.T., 2012. Microglial activation-tuning and pruning adult neurogenesis. Front Pharmacol. 3, 1-9. https://doi.org/10.3389/fphar.2012.00041

Guo, L.T., Wang, S.Q., Su, J., Xu, L.X., Ji, Z.Y., Zhang, R.Y., Zhao, Q.W., Ma, Z.Q., Deng, X.Y., Ma, S.P., 2019. Baicalin ameliorates neuroinflammation-induced depressive-like behavior through inhibition of toll-like receptor 4 expression via the PI3K/AKT/FoxO1 pathway, J Neuroinflammation. 16, 95. https://doi.org /10.1186/s12974-019-1474-8

Hsieh, C.F., Liu, C.K., Lee, C.T., Yu, L.E., Wang, J.Y., 2019. Acute glucose fluctuation impacts microglial activity, leading to inflammatory activation or self-degradation. Sci Rep. 9, 840. https://doi.org/10. 1038 /s 41598-018-37215-0

Hu, X.M., Leak, R.K., Shi, Y.J., Suenaga, J., Gao, Y.Q., Zheng, P., Chen, J., 2015. Microglial and macrophage polarization-new prospects for brain repair. Nat Rev Neurol. 11, 56-64. https://doi.org/10.1038/nrneurol. 2014. 207

Hwang, I.K., Choi, J.H., Nam, S.M., Park, O.K., Yoo, D.Y., Kim, W., Yi, S.S., Won, M.H., Seong J.K., Yoon Y.S., 2014. Activation of microglia and induction of pro-inflammatory cytokines in the hippocampus of type 2 diabetic rats. Neurol Res. 36, 824-832. https://doi.org/ 10.1179/1743132814y.0000000330 
Hwang, I.K., Yi, S.S., Kim, Y.N., Kim, I.Y., Lee, I.S., Yoon, Y.S., Seong, J.K., 2008. Reduced hippocampal cell differentiation in the subgranular zone of the dentate gyrus in a rat model of type ii diabetes. Neurochem Res. 33, 394-400. https://doi.org/10.1007/s11064-007-9440-8.

Jimeno, B., Hau, M., Verhulst, S., 2018. Corticosterone levels reflect variation in metabolic rate, independent of 'stress'. Sci Rep. 8, 13020. https://doi.org/10.1038/s41598-018-31258-Z

Jung, Y.S., Park, J.H., Kim, H., Kim, S.Y., Hwang, J.Y., Hong, K.W., Bae, S.S., Choi, B.T., Lee, S.W., Shin, H.K., 2016. Probucol inhibits LPS-induced microglia activation and ameliorates brain ischemic injury in normal and hyperlipidemic mice. Acta Pharmacologica Sinica. 37, 1031-1044. https://doi.org/10.1038/aps.2016.51

Kalkman, H.O., Feuerbach, D., 2016. Antidepressant therapies inhibit inflammation and microglial M1polarization. Pharmacol Ther, 163, 82-93. https://doi.org/10.1016/j.pharmthera.2016.04.001.

Lebedeva, K.A., Caruncho, H.J., Kalynchuk L.E., 2017. Cyclical corticosterone administration sensitizes depression-like behavior in rats. Neurosci Lett. 650, 45-51. https://doi.org/10.1016/j.neulet.2017.04.023

Lin, X.Y., Han, Y.S., Meng, P., Yang, H., Wang, Y.H., 2016. Discussion on the Traditional Chinese Medicine pathogenesis of diabetes related depression. Shizhen Guo Yi Guo Yao. 27, 1942-1943. https://doi.org/10.3969/j. issn.1008-0805.2016.08.056

Liu, J., Wang, Y.H., Li, Wei, Liu L., Yang H., Meng P., Han Y.S., 2019. Structural and functional damage to the hippocampal neurovascular unit in diabetes-related depression. Neural Regen Res. 14, 289-297. https://doi.org/10.4103/1673-5374.244794

Liu J, Wang YH, Xu YL, Meng P, Liu L, Yang H, Han YS (2016) An improved primary culture method for hippocampal neurons in fetal rat and damage of neurons subjected to diabetes mellitus with depression. Shenjing Jiepou Xue Zazhi. 32:459-465.

Liu, Y.Y., Tang, Y., Lin, C.S., Zhou, L., Xiang, L., Luo, R., Sun, X.M., Zhao, X.S., 2016. Effects of zuogui pill on kidney-yin deficiency syndrome based on theory of generating yin by activating yang. Chinese Archives of Traditional Chinese Medicine. 34, 2321-2323. https://doi.org/10.13193/j.issn.1673-7717. 2016.10.003

Liu Z., Meng P., Yang H., Zhao H.Q., Wang Y.H., 2018. Effects of Zuogui Jiangtang Jieyu prescription on pathological morphology and apoptosis of hippocampal neurons in rats of diabetes mellitus with depression. Journal of Information on TCM. 25(9), 44-48.

Marshall, S.A., McClain, J.A., Kelso, M.L., Hopkins, D.M., Pauly, J.R., Nixon, K., 2013. Microglial activation is not equivalent to neuroinflammation in alcohol-induced neurodegeneration: the importance of microglia phenotype. Neurobiol Dis. 54, 239-251. https://doi.org/ 10.1016/j.nbd.2012.12.016

Michelle, M.L., Toda, H., Palmer, T.D., 2003. Inflammatory blockade restores adult hippocampal neurogenesis. Science. 302, 1760-1764. https://doi.org/10.1126/science.1088417

Nimmerjahn, A., Kirchhoff, F., Helmchen, F., 2005. Resting microglial cells are highly dynamic surveillants of brain parenchyma in vivo. Science. 308, 1314-1318. https://doi.org/10.1126/science.1110647

Patterson, S.L., 2015. Immune dysregulation and cognitive vulnerability in the aging brain: Interactions of microglia, IL-1 $\beta$, BDNF and synaptic plasticity. Neuropharmacology. 96, 11-18. https://doi.org/ 10.1016/j. neuropharm.2014.12.020

Réus, G. Z., Fries, G.R., Stertz, L., Badawy, M., Passos, I.C., Barichello, T., Kapczinski, F., Quevedo, J., 2015. The role of inflammation and microglial activation in the pathophysiology of psychiatric disorders. Neuroscience. 300, 141-154. https://doi.org/10.1016/j.neuroscience.2015.05.018

Roy, T., Lloyd, C.E., 2012. Epidemiology of depression and diabetes: A systematic review. J Affect Disord. 142, S8-21. https://doi.org/10.1016/s0165-0327(12)70004-6

Ruan, P., Ruan H.R., 2006. Clinical study on hypericum perforatum and syndrome differentiation for depression. Shanghai Journal of Traditional Chinese Medicine. 40, 24-25. https://doi.org/10.16305/j.1007-1334.2006.10.012

Steelman, A.J., Li, J., 2014. Astrocyte galectin-9 potentiates microglial TNF secretion. J. Neuroinflammation. 11, 144. https://doi.org/10.1186/s12974-014-0144-0

Steiner, J., Walter, M., Gos, T., Guillemin, G.J., Bernstein, H.G., Sarnyai, Z., Mawrin, C., Brisch, R., Bielau, H., Meyer zu Schwabedissen, L., Bogerts, B., Myint, A.M., 2011. Severe depression is associated with increased microglial quinolinic acid in subregions of the anterior cingulate gyrus: evidence for an immune-modulated glutamatergic neurotransmission?, J. Neuroinflamm. 8, 94-102. https://doi.org/10.1186/1742-2094-8-94

Takaki, J., Fujimori, K., Miura, M., Suzuki, T., Sekino, Y., Sato, K., 2012. L-glutamate released from activated microglia downregulates astrocytic L-glutamate transporter expression in neuroinflammation: the 'collusion' hypothesis for increased extracellular L-glutamate concentration in neuroinflammation. J Neuroinflammation. 9, 275. https://doi.org/10.1186/1742-2094-9-275.

Tian, X.C., Liu, H.L., Xiang, F., Xu, L., Dong, Z., 2019. $\beta$-caryophyllene protects against ischemic stroke by promoting polarization of microglia toward M2 phenotype via the TLR4 Pathway. Life Sci. 15, 116915. https://doi.org/10.1016/j.lfs.2019.116915.

Wang, Q., 2014. Clinical investigation on antidepressant effect of curcumin. Chinese Medicine Modern Distance Education of China. 10, 145-146. https://doi.org/10.3969/j.issn.1672-2779.2014.10.089 
Wang, Y.H., 2014. Zuogui pill combined with metformin in the treatment of qi and yin deficiency type 2 diabetes research random parallel controlled study. Journal of Practical Traditional Chinese Internal Medicine. 3, 92-94. https://10.13729/j.issn.1671-7813.2014.03.47

Wang, Y.H., Yang, H., Li, W., Meng, P., Han, Y.S., Zhang, X.L., Cao, D.L., Tan, Y.S., 2015. Zuogui Jiangtang Jieyu formulation prevents hyperglycaemia and depressive-like behaviour in rats by reducing the glucocorticoid level in plasma and hippocampus. Evid Based Complement Alternat Med. 158361, 10. https://doi.org/10.1155/2015/158361.

Wang, Y.H., Yin, L.T., Yang, H., Li, X.L., Wu K.G., 2014. Hypoglycemic and anti-depressant effects of zuogui jiangtang jieyu formulation in a model of unpredictable chronic mild stress in rats with diabetes mellitus. Exp Ther Med. 8 (1), 281-285. https://doi.org/10.3892/etm.2014.1681

Xiang Y., Wu M.Y., Zhao H.Q., Liu J., Lei C., Meng P., Zhang X.L., Ling J., Wang Y.H., 2020. Effects of Zuogui Jiangtang Jieyu Fang on NR and mEPSC in hippocampal neurons of fetal rat with diabetes mellitus complicated with depression. JBUTCM. 40(3), 274-278.

Xie, W., Cai, L, Yu, Y., Gao, L., Xiao, L., He, Q., Ren, Z., Liu Y., 2014. Activation of brain indoleamine 2,3dioxygenase contributes to epilepsy-associated depressive-like behavior in rats with chronic temporal lobe epilepsy, J. Neuroinflamm. 11, 41-50. https://doi.org/10.1186/1742-2094-11-41

Yang, Z.Y., Liu, B.P., Yang, L.E., Zhang, C., 2019. Platycodigenin as potential drug candidate for Alzheimer's disease via modulating microglial polarization and neurite regeneration. Molecules, 24, 3207-3220. https:// doi.org /10.3390/molecules24183207

Yang, Y.X., Ye, Y.Q., Kong, C.G., Su, X.H., Zhang, X., Bai, W., He, X.S., 2019. MiR-124 Enriched exosomes promoted the M2 polarization of microglia and enhanced hippocampus neurogenesis after traumatic brain injury by inhibiting TLR4 pathway. Neurochem Res. 44, 811-828. https://doi.org/10.1007/s11064-01802714-z.

Zanoveli, J.M., Morais, H.d., Dias, I.C., Schreiber, A.K., Souza, C.P., Cunha, J.M., 2016. Depression associated with diabetes: From pathophysiology to treatment. Curr Diabetes Rev. 12, 165-178. https://doi.org/ 10.2174/1573399811666150515125349.

Zhao, Q.Y., Wu, X.H., Yan, S., Xie, X.F., Fan, Y.H., Zhang, J.Q., Peng, C., You, Z.L., 2016. The antidepressant -like effects of pioglitazone in a chronic mild stress mouse model are associated with PPAR $\gamma$-mediated alteration of microglial activation phenotypes. J Neuroinflammation. 13, 259. https://doi.org/10.1186/s12974 $-016-0728-y$

Zhang, L., Previn, R., Lu, L., Liao, R.F., Jin, Y., Wang, R.K., 2018. Crocin, a natural product attenuates lipopolysaccharide-induced anxiety and depressive-like behaviors through suppressing NF-kB and NLRP3 signaling pathway. Brain Res Bull. 142, 352-359. https://doi.org/10.1016/j.brainresbull.2018.08.021

Zhang, X., Dong, H.Q., Zhang, S.S., Lu S.M., Sun, J., Qian, Y.N., 2015. Enhancement of LPS-induced microglial inflammation response via TLR4 under high glucose conditions. Cell Physiol Biochem. 35, 1571-1581. https://doi.org/10.1159/000373972

Zhang, X.L., Meng, P., Wang Y.H., Xiang Y., Liu, L., Han Y.S., 2015. Effects of baishile capsules on neurogenesis related protein in hippocampal DG and CA3 area of model rats with chronic unpredicted mile stress depression. Chinese Journal of Information on TCM. 22(5), 52-56.

Zhang, X.L., Wang, Y.H., Yang, H., Xu Y.L., 2014. Protective effects of Zuoglli Jiangtang Jieyu Fang on rat hippocampal neuron cells. JBUTCM. 34, 8-12.

Zhang, X.L., Wang, Y.H., Yang H., Meng P., Tan X.W., Chai S., 2015. Effects of zuogui jiangtang jieyu formulation on the learning and memory abilities and ultrastructure of hippocampus in diabetes mellitus rats with depression. Shizhen Guo Yi Guo Yao. 26, 787-789. 


\section{Figures}

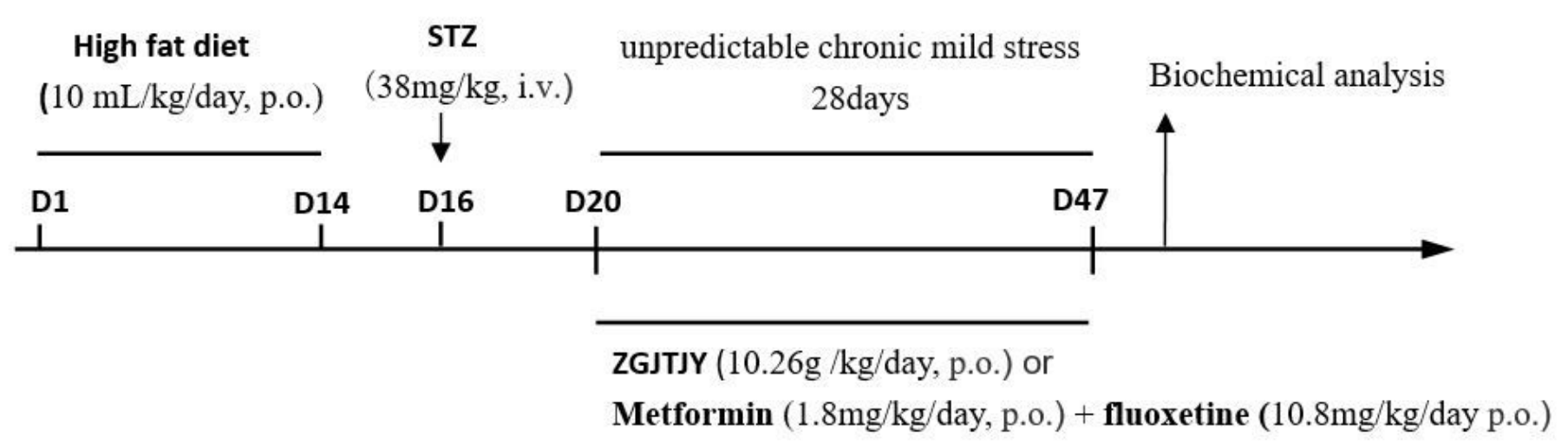

Fig. 1.

\section{Figure 1}

The flow chart of the study protocol

C A 1
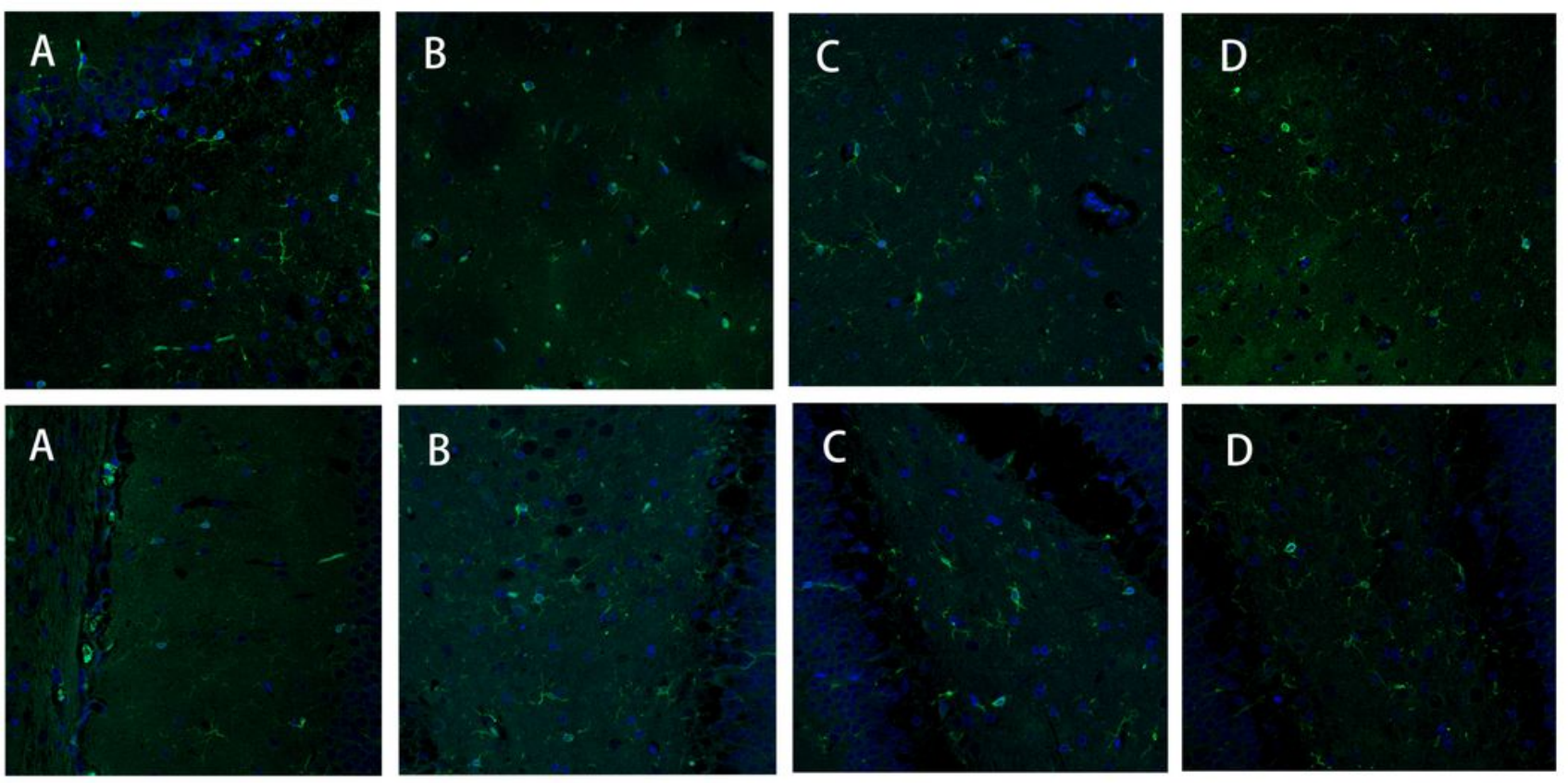

Figure 2

Immunofluorescence staining of Iba1 (green) to detect microglial activation in the hippocampal CA1 and DG areas, with DAPI staining is shown in blue $(200 \mathrm{x})$. "Resting" microglia in the control group. (A) The vehicle group showed activated microglia with cell expansion, thicker branching, and a decreased cell number (B). DMGB/F reversed abnormalities in the CA1, but not DG, area. (C) ZGJTJY reversed these abnormalities in both the CA1 and DG areas (D), $n=3$. 
A
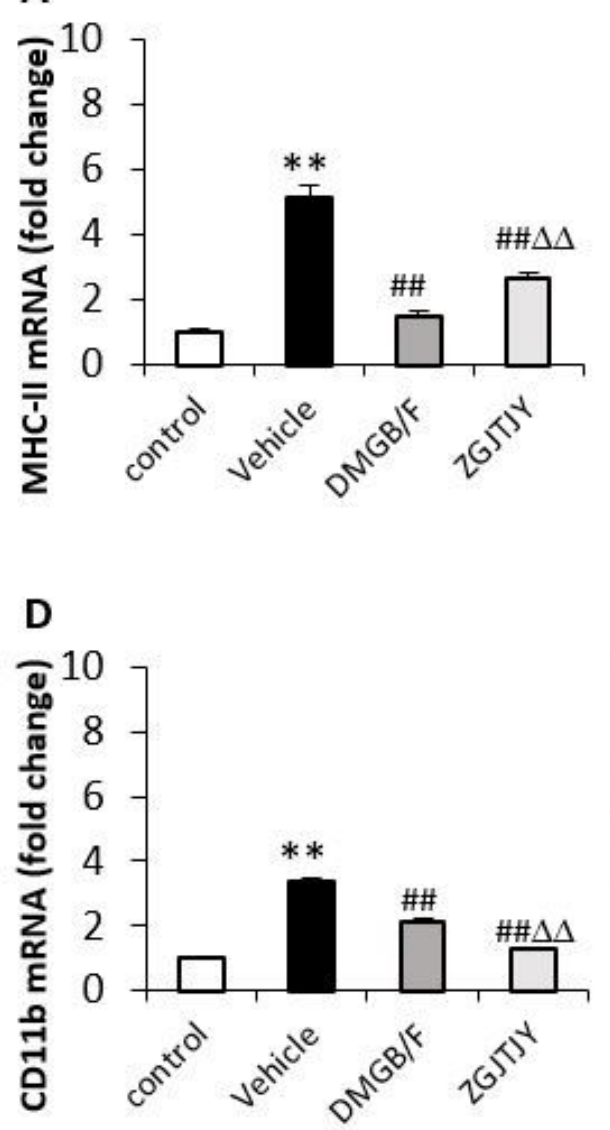
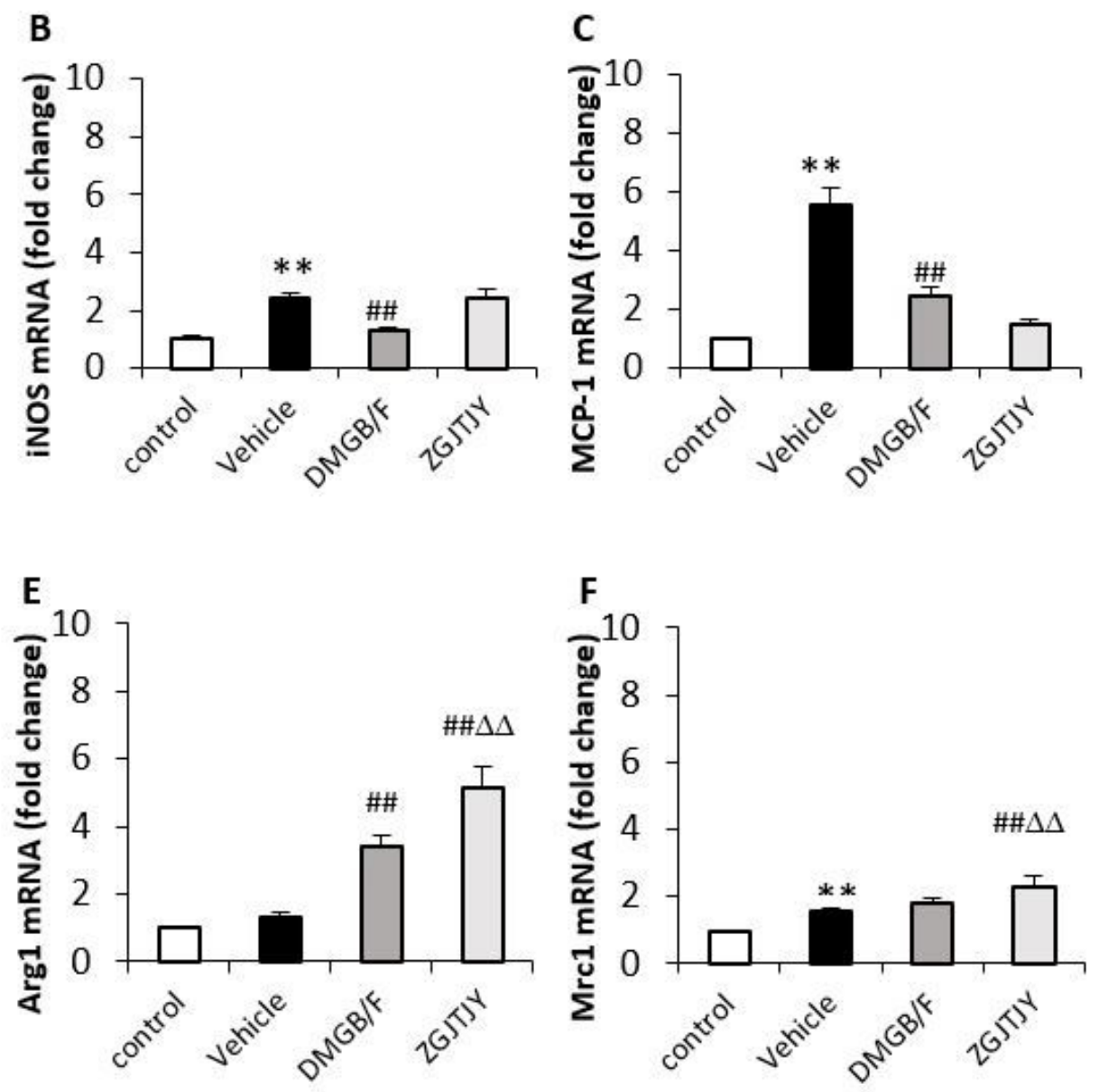
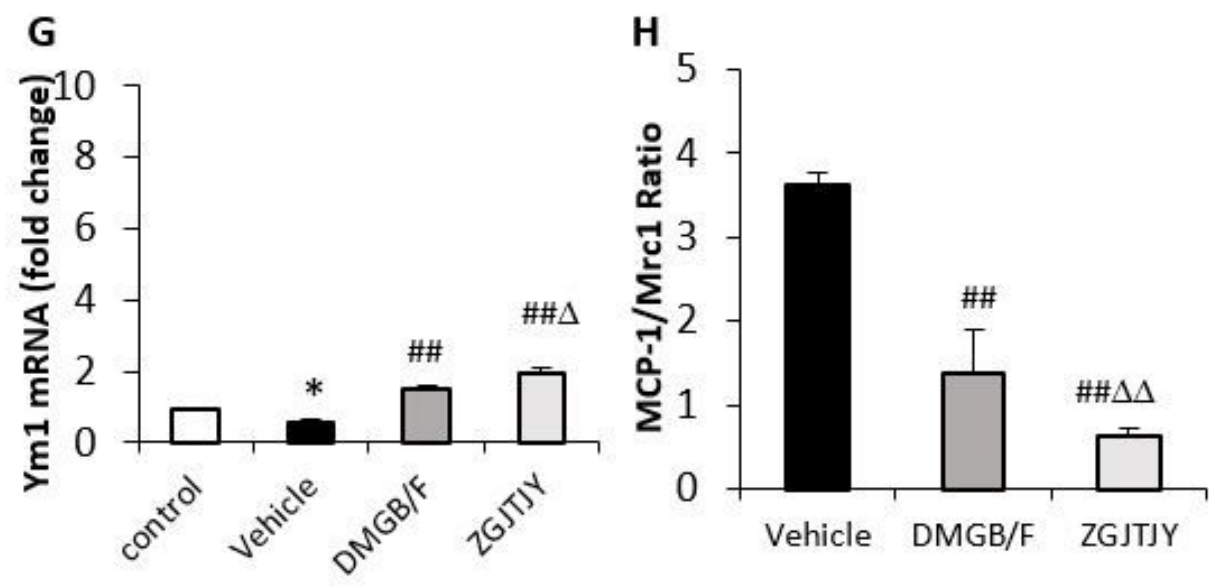

Fig. 3

Figure 3

mRNA expressions of hippocampal M1/M2 microglia markers. Fold increase of M1 markers (MHC区, iNOS, MCP-1, CD11b) (A, B, C, D); Fold increase of M2 microglia markers (Arg1, Mrc1, Ym1) (E, F, G); Fold increase of the ratio of MCP-1/Mrc1 $(\mathrm{H})$. Each bar represents the mean $\pm S D$ of three samples. ${ }^{*} P<0.05$ and ${ }^{* * P}<0.01$, significantly different from the control group. $\# \mathrm{P}<0.05$ and \#\#P<0.01, significantly different from the vehicle group. $\Delta \mathrm{P}<0.05$ and $\Delta \Delta \mathrm{P}<0.01$, significantly different from the $\mathrm{DMGB} / \mathrm{F}$ group. 

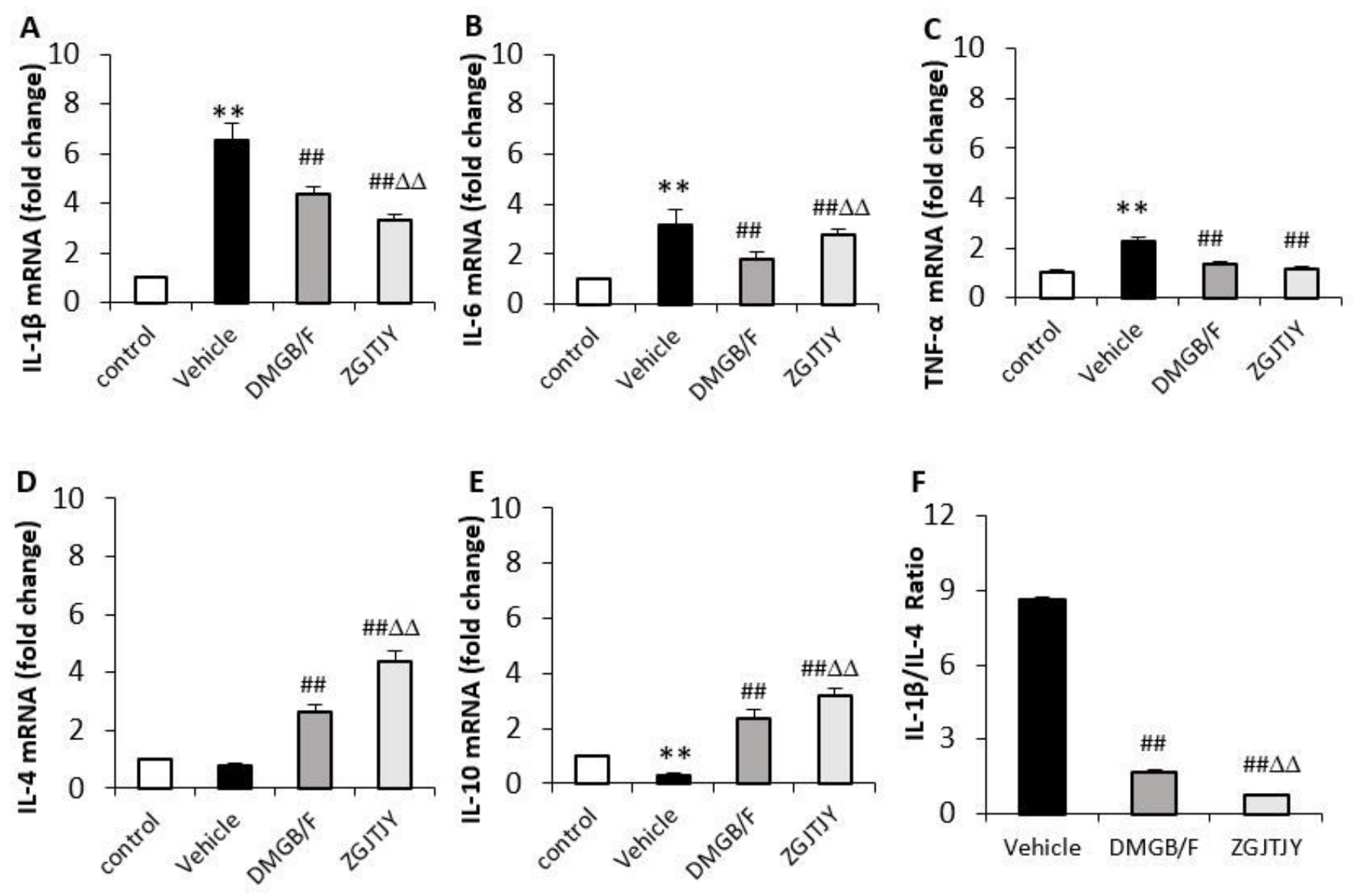

Fig. 4

Figure 4

mRNA expressions of different hippocampal inflammatory cytokines. Fold increase of pro-inflammatory

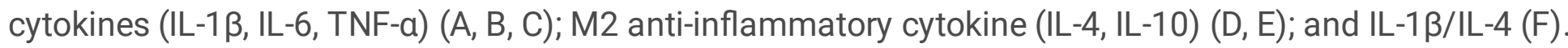
Each bar represents the mean \pm SD of three samples. ${ }^{*} P<0.05$ and ${ }^{*} P<<0.01$, significantly different from the control group. $\# P<0.05$ and $\# \# P<0.01$, significantly different from the vehicle group. $\triangle P<0.05$ and $\triangle \triangle P<0.01$, significantly different from the DMGB/F group. 

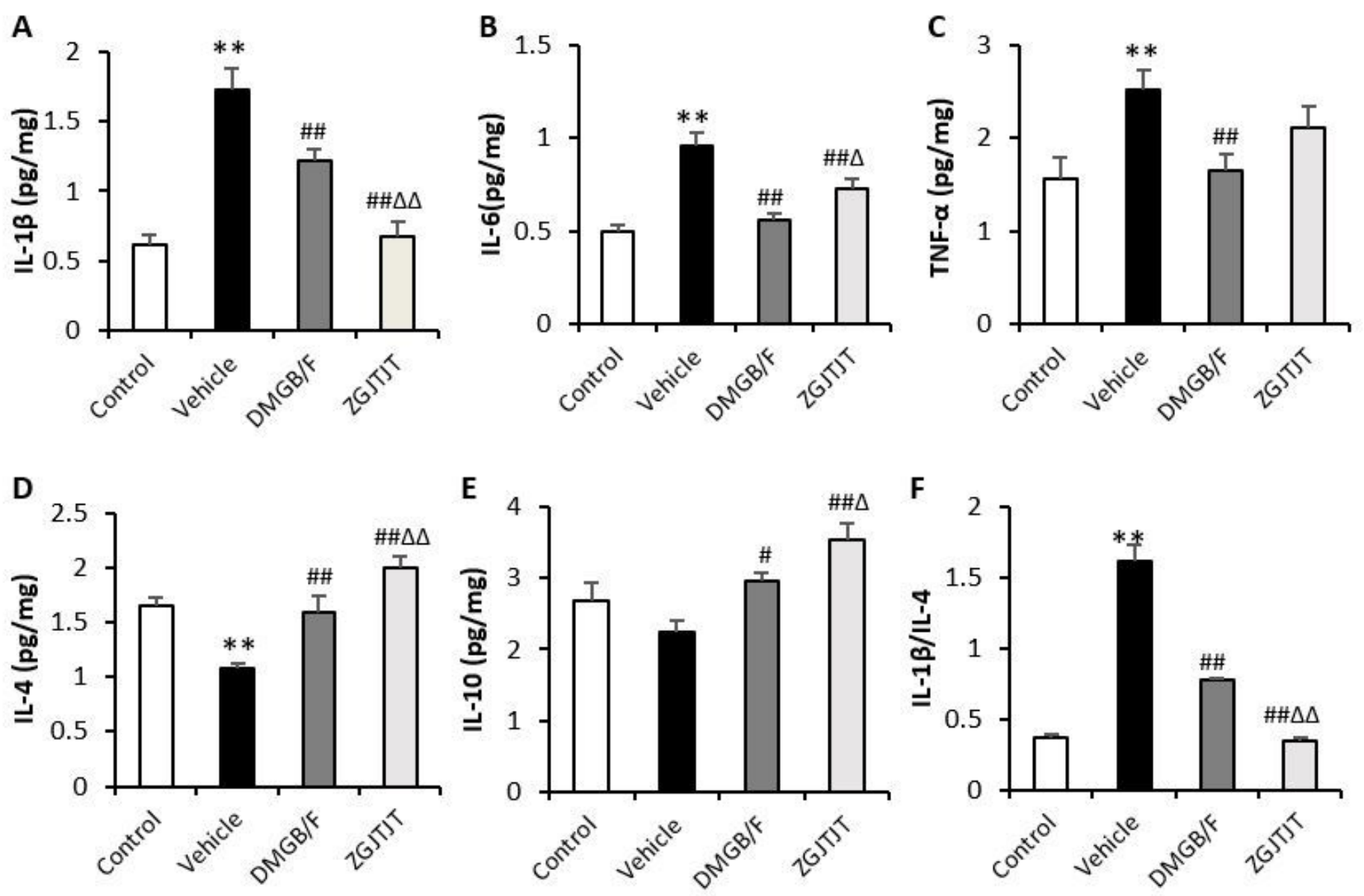

Fig. 5 .

\section{Figure 5}

ELISA analysis of different hippocampal inflammatory cytokines. Levels of M1 pro-inflammatory (IL-1 IL-6, TNF-a) (A, B, C) and anti-inflammatory cytokines (IL-4, IL-10) (D, E). The ratio of IL-1 $\beta /$ IL-4 (F). Each bar represents the mean $\pm S D$ of three samples. $\# P<0.05$ and \#\#P $<0.01$, significantly different from the vehicle group. $\Delta \mathrm{P}<0.05$ and $\Delta \Delta \mathrm{P}<0.01$, significantly different from the $\mathrm{DMGB} / \mathrm{F}$ group.
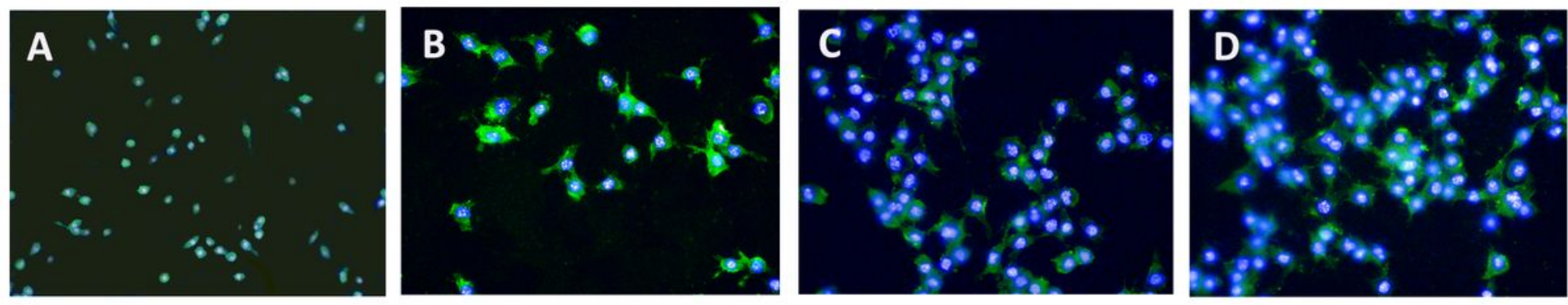

Figure 6

Immunofluorescence staining of Iba1(green) for detecting microglial activation induced by high glucose and corticosterone, with DAPI staining shown in blue(200x). HAPI cells in the control group (A). Activated 
microglia with cell expansion, thickening of branching, and decreased cell number in the Vehicle group (B). DMGB/F- (C) and ZGJTJY-containing serum(D) reversed these abnormalities.
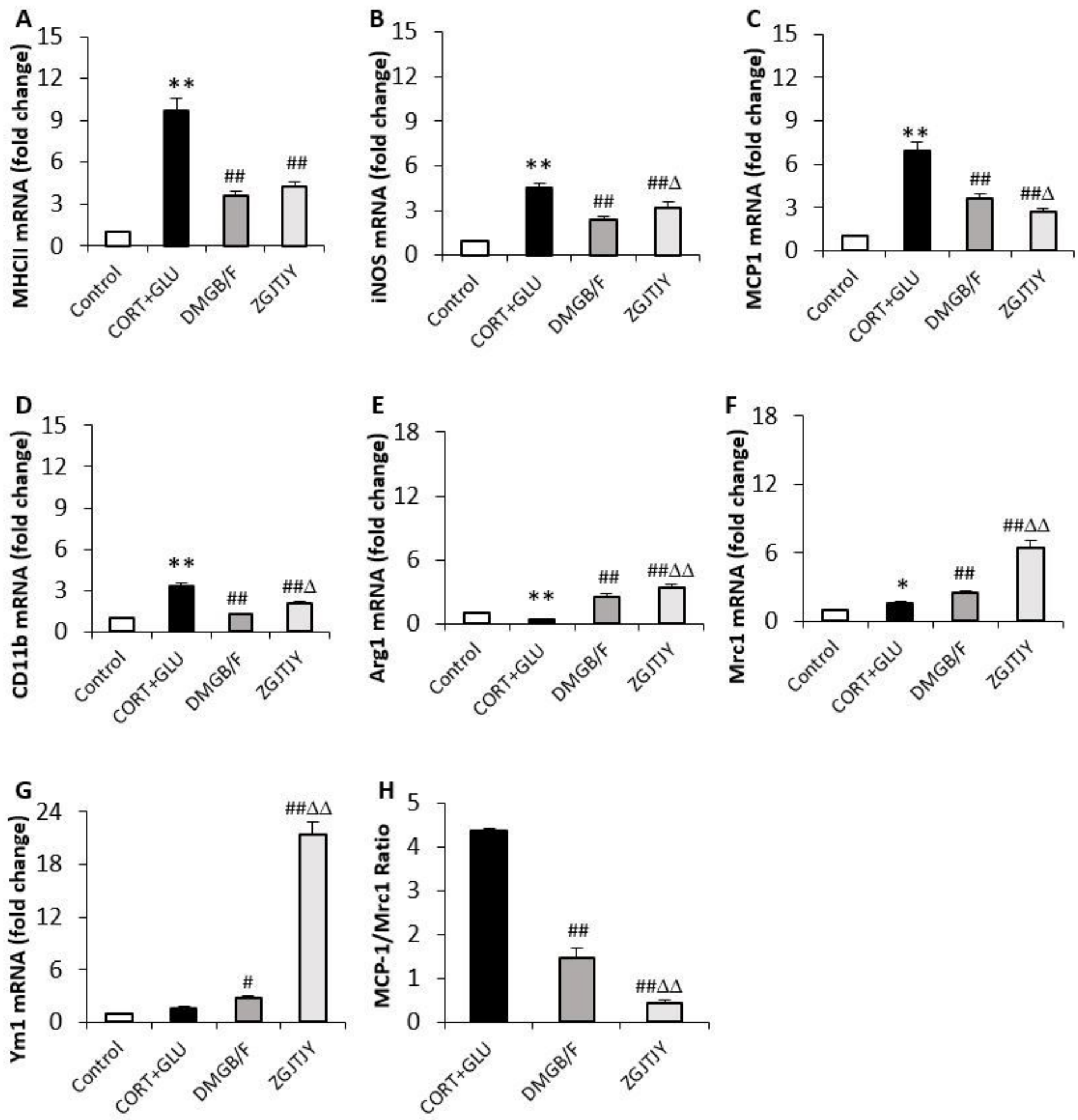

Fig. 7

Figure 7

mRNA expressions of M1/M2 microglia markers in HAPI cells. Fold increase of M1 markers (MHC区, iNOS, MCP-1, CD11b) (A, B, C, D); M2 microglia markers (Arg1, Mrc1, Ym1) (E, F, G); and MCP-1/Mrc1 ratio (H). Each bar represents the mean $\pm S D$ of three wells. ${ }^{*} P<0.05$ and ${ }^{*} P P<0.01$, significantly different from 
the control group. \#P $<0.05$ and \#\#P $<0.01$, significantly different from the vehicle group. $\Delta P<0.05$ and $\Delta \triangle \mathrm{P}<0.01$, significantly different from the $\mathrm{DMGB} / \mathrm{F}$ group.
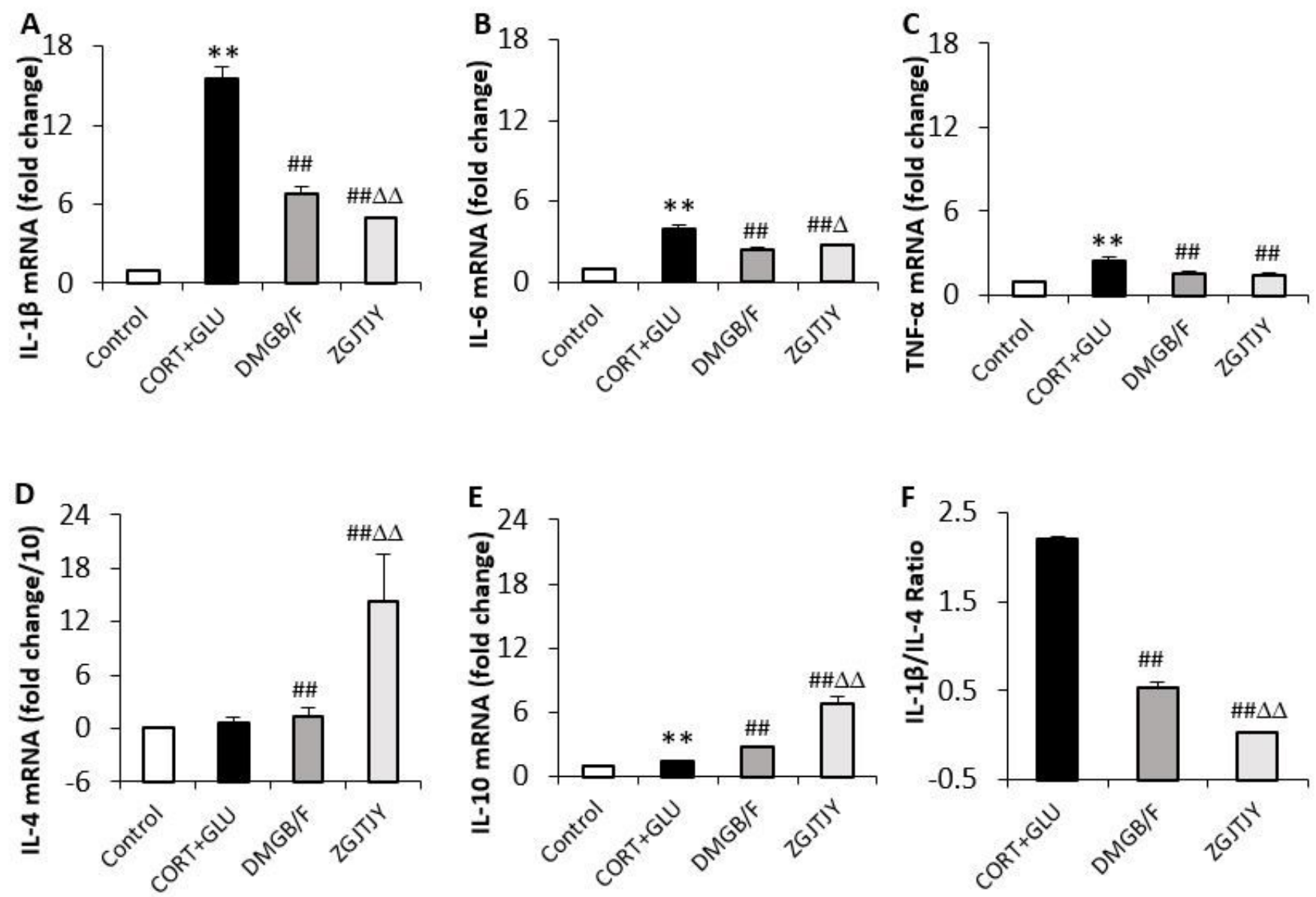

Fig. 8

\section{Figure 8}

mRNA expressions of different inflammatory cytokines in HAPI cells. Fold increase of pro-inflammatory cytokines (IL-1 $\beta$, IL-6, TNF-a) (A, B, C); M2 anti-inflammatory cytokine (IL-4, IL-10) (D, E); and IL-1 $\beta / I L-4$ ratio $(F)$. Each bar represents the mean $\pm S D$ of three wells. ${ }^{*} \mathrm{P}<0.05$ and ${ }^{*} \mathrm{P}<0.01$, significantly different from the control group. \#P $<0.05$ and \#\#P $<0.01$, significantly different from the vehicle group. $\Delta P<0.05$ and $\Delta \Delta P<0.01$, significantly different from the $D M G B / F$ group. 

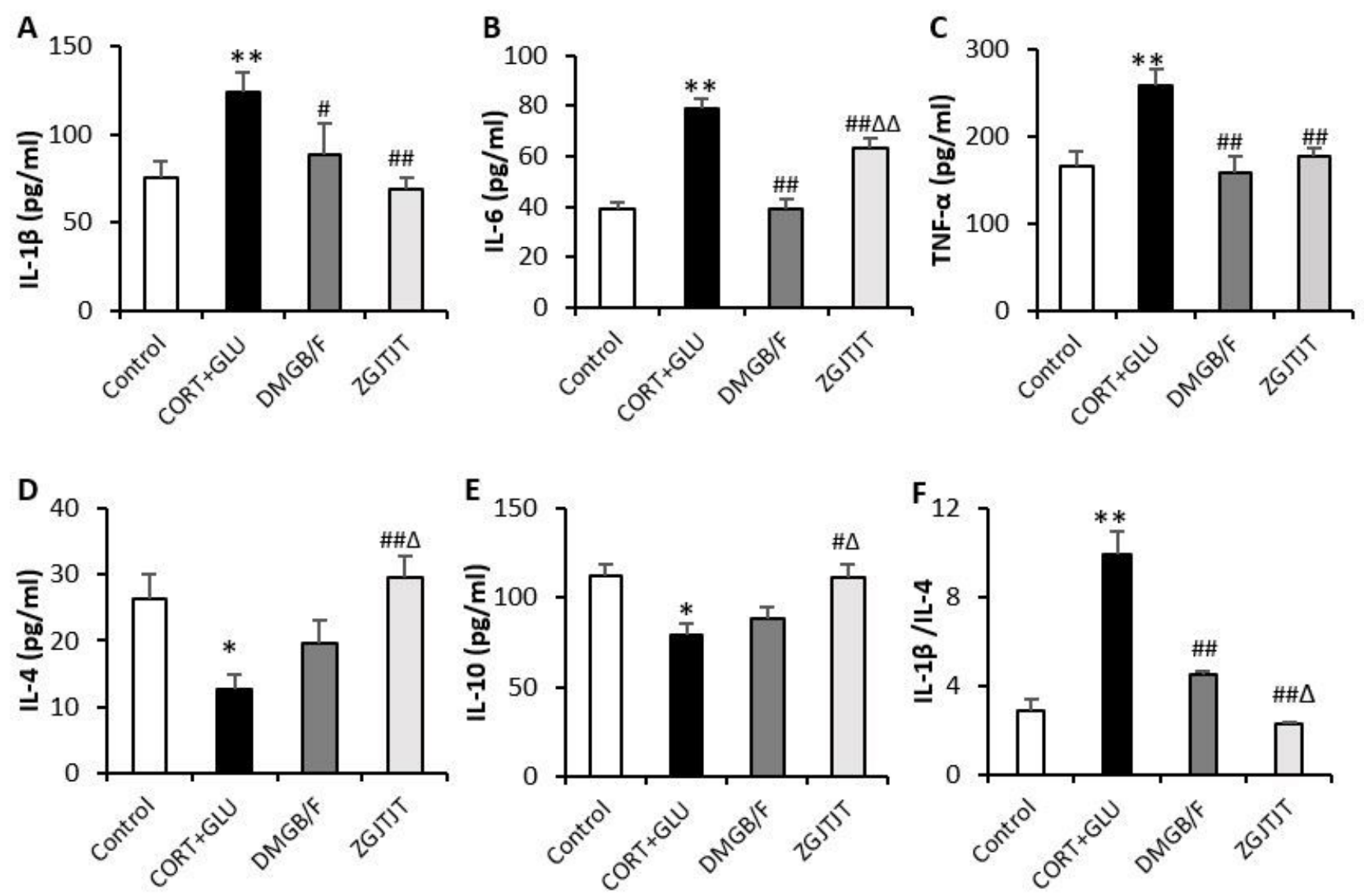

Fig. 9

Figure 9

ELISA analysis of different inflammatory cytokines in cultured HAPI cell supernatant. Content of proinflammatory (IL-1 $\beta, I L-6, T N F-a)(A, B, C)$ and M2 anti-inflammatory cytokines (IL-4, IL-10) (D, E). The ratio of IL-1 $\beta / I L-4(F)$. Each bar represents the mean $\pm S D$ of three wells. \#P $<0.05$ and \#\#P $<0.01$, significantly different from the vehicle group. $\Delta P<0.05$ and $\Delta \Delta P<0.01$, significantly different from the DMGB/F group. 